\title{
A New Method of Treating Light-Scattering Data on Dilute Polymer Solutions
}

\author{
Hiroshi Fujita \\ Department of Polymer Science, Osaka University, Toyonaka, Japan.
}

(Received June 2, 1970)

\begin{abstract}
The square-root method of treating light-scattering data on narrowdistribution polymers in dilute solution ceases to be effective for samples of very high molecular weight in good solvent unless the experimental data are obtained down to a fairly small scattering angle (of the order of $10^{\circ}$ ). This communication reports the development of a new method which is expected to be particularly useful for such polymer-solvent systems, on the basis of recent experimental evidence that the particle scattering factor $\boldsymbol{P}(\theta)$ is little affected by the long-range interference between chain segments and follows quite closely the Debye equation for Gaussian chains. The potentiality of the method is demonstrated with data on polyisobutylene in cyclohexane and those on $\operatorname{poly}(\alpha$-methylstyrene) in benzene.

KEY WORDS Light Scattering/Square-Root Method/Debye Equation for Particle-Scattering Factor / Monodisperse Polymer / Polydisperse Polymer /
\end{abstract}

The availability of nearly monodisperse or very narrow-distribution samples of polymer in recent years has stimulated experimentalists to undertake precise measurements which purported to make unambiguous tests of a variety of theories so far presented on dilute polymer solutions. Thus light-scattering measurements ${ }^{1}$ with such samples have revealed in the plots of $K c / R(\theta)$ at $c=0$ against $\sin ^{2}(\theta / 2)$ an upward curvature which had been expected from the theory of Gaussian coils ${ }^{2}$ but had not been detected clearly by measurements of previous workers who dealt with broad-distribution samples of polymer. Here $c$ is the polymer concentration, $R(\theta)$ is the excess Rayleigh ratio of the solution, and $K$ is the familiar light-scattering factor. The appearance of such a curvature gives rise to the difficulty of extrapolating the plot for the purpose of determining the molecular weight $M$ and the mean-square radius of gyration $\left\langle S^{2}\right\rangle$ of the dissolved solute from its ordinate intercept and initial slope.

Berry $^{3}$ showed that this difficulty is largely circumvented if the square root of $K c / R(\theta)$ is plotted against $\sin ^{2}(\theta / 2)$ and applied this method to his data on samples of anionically polymerized polystyrene. Norisuye, et al. ${ }^{4}$ enjoyed its merit in a study with sharply fractionated samples of polychloroprene. It appears that the square-root method of Berry is now becoming a standard procedure of treating light-scattering data on narrow-distribution samples of polymers. However, in his recent measurements with anionically polymerized $\operatorname{poly}\left(\alpha\right.$-methylstyrene) Utiyama ${ }^{5}$ has shown that the square-root method is of limited value for a sample of as high an $M$ as a few million in good solvent, provided that the measurement of $R(\theta)$ cannot be extended down to $\theta$ below $35^{\circ}$, as is the case with most of the commercially available light-scattering photometers. This fact is illustrated in Figure 1, where his data ${ }^{6}$ on a sample having an $M$ of about seven million in benzene are plotted in accordance with the square-root method. It is seen that the availability of data points for $\theta$ below $30^{\circ}$ is almost imperative in order to evaluate the correct intercept and initial slope of the plot and hence of $M$ and $\left\langle S^{2}\right\rangle$ of this system.

In recent years, there has been considerable interest in designing a light-scattering photometer by which one may extend the measurement of $R(\theta)$ down to $\theta$ much smaller than $35^{\circ}$, and several kinds of "low-angle" photometer have been reported..$^{1,6-9}$ Even with such achievements 


\section{H. FujitA}

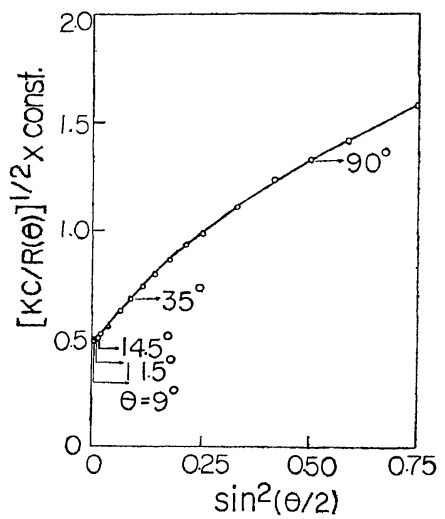

Figure 1. Utiyama's data, plotted in accordance with the square-root method, for a sample of anionically prepared poly ( $\alpha$-methylstyrene $)$ in benzene at $30^{\circ} \mathrm{C}$ (note that the measurements were made down to $\theta=9^{\circ}$ ).

in the instrumentation, however, one must keep in mind that light-scattering measurements at low scattering angles are still a task of considerable difficulty, being liable to be affected by various disturbing factors.

Summarizing the present situation, one may wish to devise a new procedure which permits one to determine $M$ and $\left\langle S^{2}\right\rangle$ correctly even under the condition where the square-root method ceases to be effective unless data are obtainable with precision down to a very low scattering angle. This paper is concerned with proposing a method relevant to this aim.

\section{THEORY}

To begin with, one defines the quantities $x$ and $y$ by

$$
\begin{gathered}
x=\left(\frac{4 \pi}{\lambda}\right)^{2}\left\langle S^{2}\right\rangle \sin ^{2} \frac{\theta}{2} \\
y=\lim _{c \rightarrow 0} \frac{R(\theta)}{K c}
\end{gathered}
$$

where $\lambda$ is the wavelength of light in the solvent medium considered. The quantity $y$ is related to the particle scattering factor $P(\theta)$ by

$$
y=M P(\theta)
$$

The theoretical range of $\theta$ is from 0 to $180^{\circ}$, but the data for $y$ are usually obtainable for $\theta$ between 35 and $150^{\circ}$.

\section{Monodisperse Polymer}

First, one considers a perfectly monodisperse polymer dissolved in a theta (or ideal) solvent. For this system the function $P(\theta)$ is represented by the Debye equation, ${ }^{2}$ and this fact gives

$$
y x^{2}=2 M\left(e^{-x}-1+x\right)
$$

Integrating from $x=0$ to $x=x$ leads to

$$
\int_{0}^{x} y x^{2} \mathrm{~d} x=2 M\left(1-e^{-x}-x+\frac{x^{2}}{2}\right)
$$

Combining these two equations yields

$$
\frac{1}{y}=\frac{1}{M}+\frac{1}{M y x^{2}} \int_{0}^{x} y x^{2} \mathrm{~d} x
$$

This may be rewritten as follows:

$$
\frac{1}{y(u)}=\frac{1}{M}+b Z(u)
$$

where

$$
b=\frac{32 \pi^{2}}{3 \lambda^{2}} \frac{\left\langle S^{2}\right\rangle}{M}
$$

and

$$
Z(u)=\left[y(u) u^{4 / 3}\right]^{-1} \int_{0}^{u} y(u) u \mathrm{~d} u
$$

with $u$ defined by

$$
u=\sin ^{3} \frac{\theta}{2}
$$

With $y$ given experimentally for a series of $\theta$ and hence of $u$, one may calculate $Z$ as a function of $u$; the integration involved may be effected by a suitable graphical or numerical method. In order for this integration to be made accurately, it is preferable that the data for $y$ are given down to as low a scattering angle as possible. However, as illustrated below, in practice, the data down to $\theta=35^{\circ}$ are sufficient; no significant error is introduced if the integrand $y(u) u$ for $u$ below $\theta=35^{\circ}$ is extrapolated rather arbitrarily from the data for higher scattering angles.

Now, with the numerical values obtained for $Z(u)$, one can plot $1 / y(u)$ against $Z(u)$ corresponding to the same $u$. According to eq 7, the resulting plots should follow a straight line whose intercept and slope are equal to $1 / M$ and $b$, respectively. The quantity $b$ is related to $\left\langle S^{2}\right\rangle$ by eq 8 . Thus the problem of evaluating $M$ and $\left\langle S^{2}\right\rangle$ of a monodisperse polymer in an 


\section{Light Scattering}

ideal solvent is reduced to drawing a straight line through $1 / y(u)$ plotted against $Z(u)$.

For a monodisperse sample of polymer dissolved in a nonideal solvent one may also plot $1 / y(u)$ against $Z(u)$, but one can no longer expect the resulting plots to follow a straight line, since the function $P(\theta)$ for this case should deviate from the Debye equation 4 due to the presence of long-range interactions between chain segments. In fact, it appears to have been long conceived implicitly that such interactions should considerably affect the form of $P(\theta)$. Importantly, it has been demonstrated quite recently by several investigators $^{1,10-13}$ that this concept must be altered. Thus it was shown that observed values of $\boldsymbol{P}(\theta)$ for narrow-distribution samples of typical polymers in good solvents followed the Debye equation to a surprisingly close degree except in the region of fairly large values of $x$. If this is generally the case, one can expect that the plot of $1 / y(u) v s ., Z(u)$ for a narrow-distribution polymer may show no appreciable curvature even when the solvent is markedly nonideal and there will be no essential difficulty in extrapolating it to $Z=0$.

It is well known from the general theory of light scattering that $1 / y$ for a monodisperse polymer in a single solvent (either ideal or nonideal) can be expanded in powers of $\sin ^{2}(\theta / 2)$ to give

$$
\frac{1}{y}=\frac{1}{M}+\frac{16 \pi^{2}}{3 \lambda^{2} M}\left\langle S^{2}\right\rangle \sin ^{2} \frac{\theta}{2}+\cdots
$$

Substituting this into eq 9 followed by integration leads to

$$
Z(u)=\frac{1}{2} \sin ^{2} \frac{\theta}{2}+\cdots
$$

From eq 11 and 12 it follows that

$$
\frac{1}{y(u)}=\frac{1}{M}+b Z(u)+\text { higher terms in } Z(u)
$$

where eq 8 has been used. From eq 13 one finds that the values of $M$ and $\left\langle S^{2}\right\rangle$ for a monodisperse sample of polymer in a given solvent, either ideal or nonideal, can be determined from the intercept and initial slope of $1 / y(u)$ plotted against $Z(u)$. Eq 7 valid for ideal solvents is a special case of eq 13, in which the plot becomes linear over the entire range of $Z$. It is encouraging for this method of determining $M$ and $\left\langle S^{2}\right\rangle$ that for the reason mentioned above the plot may be extrapolated to $Z=0$ with no essential difficulty even when the data are obtained with nonideal solvents.

\section{Polydisperse Polymer}

For a polydisperse sample of polymer dissolved in a single solvent it can be shown that, in place of eq 13, the following expansion holds. irrespective of the nonideality of the solvent

$$
\frac{1}{y(u)}=\frac{1}{M_{w}}+b_{z w} Z(u)+\text { higher terms in } Z
$$

Here $M_{w}$ denotes the weight-average molecular weight of the sample, and $b_{z w}$ is defined by

$$
b_{z w}=\frac{32 \pi^{2}}{3 \lambda^{2}} \frac{\left\langle S^{2}\right\rangle_{z}}{M_{w}}
$$

with $\left\langle S^{2}\right\rangle_{z}$ being the $z$-average mean-square radius. of gyration. By the same argument as above one can conclude from eq 14 that $M_{w}$ and $\left\langle S^{2}\right\rangle_{z}$ of a polydisperse sample of polymer may be evaluated from the intercept and initial slope of $1 / y(u)$ plotted against $Z(u)$. However, in this case, nothing general can be argued in advance about the curvature of the plot. The molecularweight distribution of the sample may sensitively manifest itself to the extent of a deviation of the plot from linearity. Thus it depends on the degree of polydispersity whether the necessary intercept and initial slope can be extrapolated with precision when this method is applied to a polydisperse sample.

\section{APPLICATIONS}

\section{Polyisobutylene in Cyclohexane}

Matsumoto ${ }^{14}$ prepared a series of very narrowdistribution samples $\left(M_{w} / M_{n} \approx 1.1\right)$ of polyisobutylene by repeated fractionations including those by a theta column, and performed careful light-scattering measurements on them in various solvents by making use of a Type PG-21 Shimazu photometer. His data on a sample having $M$ of about three million in cyclohexane at $25^{\circ} \mathrm{C}$ are chosen here for illustration of the method presented above.

Figure 2 shows experimental values of $y(u) u$ plotted against $u$. The solid line that fits the plotted points has been drawn for graphical 


\section{H. FuIITA}

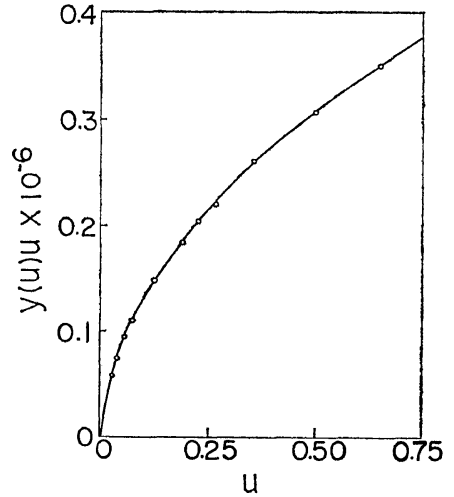

Figure 2. Plots of $y(u) u$ against $u$ prepared from Matsumoto's data for a narrow-distribution sample of polyisobutylene in cyclohexane at $25^{\circ} \mathrm{C}$.

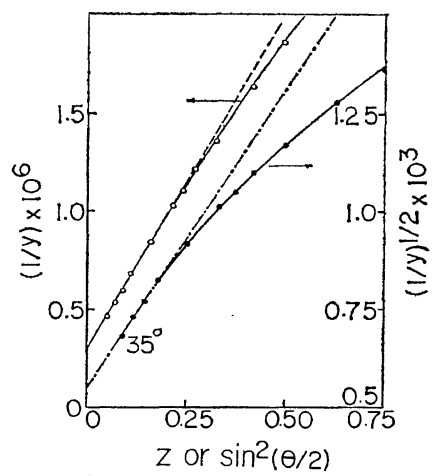

Figure 3. Tests of the present method (open circles) and the square-root method (closed circles) with Matsumoto's data on polyisobutylene quoted in Figure 2 (see eq 9 for the definition of $Z$ ).

evaluation of the integral involved in $Z(u)$. With the photometer used the smallest scattering angle down to which reliable measurements could be made was $35^{\circ}$. The left-most open circle in Figure 2 corresponds to this angle. One can see that $y(u) u$ for $u$ below this point can be extrapolated rather uniquely from the data points for the higher angles. A small error in this extrapolation is immaterial, making no significant contribution to $Z(u)$. This feature is one of the merits of the present method.

Figure 3 shows the data plotted in accordance with the present method (open circles) and with the square-root method of Berry (closed circles). Note that the ordinate scale for the closed circles is taken twice as large as that for the open circles. The advantage of the present method over the square-root method is obvious from the graph. The line followed by the open circles has a curvature much smaller than that followed by the closed circles, and can be extrapolated to $Z=0$ with no uncertainty. The dashed line indicated may be taken as the correct initial tangent to this line, giving for the polymersolvent system considered

$$
M_{w}=3.3_{3} \times 10^{6}, \quad\left\langle S^{2}\right\rangle_{z}=0.96_{6} \times 10^{-10} \mathrm{~cm}^{2}
$$

However, in this case, the situation with the square-root plot is not too bad, where one can see that the first four points in the region of small $\theta$ arrange themselves well on a straight line and enable one to determine the desired intercept and initial slope of the plot with fair confidence. The chain line in the figure has been drawn in such a manner that one may obtain the above-indicated values of $M_{w}$ and $\left\langle S^{2}\right\rangle_{z}$ from the square-root plot.

The curvature of the line fitting the open circles in the region of high scattering angles, though small, is unmistakable. It may be due both to the strong nonideality of the system (cyclohexane is known to be a very good solvent for polyisobutylene) and to a polydispersity of the sample. At present, nothing can be argued about which of these effects is mainly responsible for the phenomenon.

\section{Poly( $\alpha$-Methylstyrene $)$ in Benzene}

The data of Utiyama ${ }^{6}$ on a sample of anionically polymerized poly ( $\alpha$-methylstyrene) in benzene at $30^{\circ} \mathrm{C}$, shown in Figure 1 for an illustration of the problematic point of the square-root method, may be taken as a good example for a test of the present method.

The open and closed circles in Figure 4 show Utiyama's data plotted in accordance with the present method and the square-root method, respectively. Actually, the closed circles are the reproduction of Figure 1. In Figure 5 is the magnified behavior of these plots in the region of relatively small scattering angles.

The situation here clearly favors the present method over the square-root method. The line fitting the open circles is accurately linear over a range up to $\theta=70^{\circ}$ at least, yielding with precision the following values for $M_{w}$ and $\left\langle S^{2}\right\rangle_{z}$ 


\section{Light Scattering}

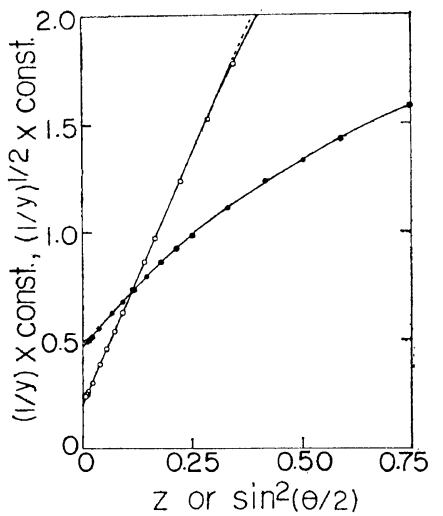

Figure 4. Utiyama's data on poly ( $\alpha$-methylstyrene) (as in Figure 1) plotted in accordance with the present method (open circles) and the square-root method (closed circles).

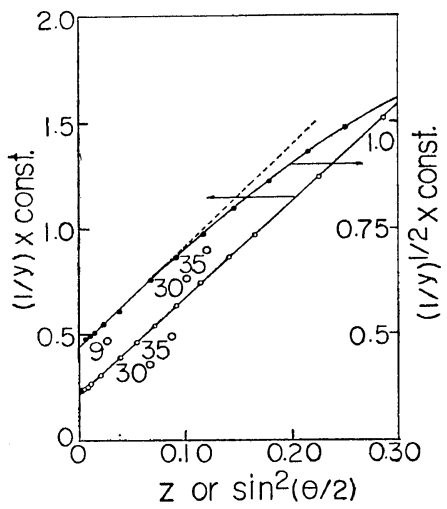

Figure 5. Showing the plots of Figure 4 with the abscissa magnified by a factor 2.5 .

of this polymer-solvent system:

$$
M_{w}=6.9_{8} \times 10^{6}, \quad\left\langle S^{2}\right\rangle_{z}=1.6_{2} \times 10^{-10} \mathrm{~cm}^{2}
$$

It should be noted that, with the present method, these results could be obtained even if the actual data were available only for $\theta$ above $30^{\circ}$. The square-root plots, here being available down to a very small scattering angle, can also be extrapolated to zero scattering angle with no ambiguity, yielding

$$
M_{w}=6.7_{1} \times 10^{6}, \quad\left\langle S^{2}\right\rangle_{z}=1.5_{8} \times 10^{-10} \mathrm{~cm}^{2}
$$

if the initial tangent is drawn as shown by the dashed line in Figure 5. However, the point of importance here is that the line fitting the closed circles exhibits a marked curvature for $\theta$ above $35^{\circ}$ so that one could not reach these numerical results if the measurements were confined to such $\theta$ only.

The two examples discussed above would suffice to demonstrate the potentiality of the present method for treating light-scattering data on narrow-distribution samples of polymer of very high molecular weight in good solvents. The difficulty of extrapolation which often troubles one in dealing with such data in terms of the square-root method is here largely removed. The only disadvantage of the present method is that the evaluation of the integral involved in $Z$ is somewhat time-consuming.

Acknowledgment. Thanks are due to Mr. T. Ueda of this laboratory for his aid in the numerical calculations, and also to Dr. H. Utiyama of Kyoto University who kindly put his unpublished data at our disposal.

\section{REFERENCES}

1. For example, H. Utiyama, N. Sugi, M. Kurata, and M. Tamura, Bull. Inst. Chem. Res., Kyoto Univ., 46, 198 (1968).

2. P. Debye, J. Phys. Colloid Chem., 51, 18 (1947).

3. G. C. Berry, J. Chem. Phys., 44, 4550 (1966).

4. T. Norisuye, K. Kawahara, A. Teramoto, and H. Fujita, J. Chem. Phys., 49, 4330 (1968).

5. H. Utiyama and Y. Tsunashima, to be published in J. Chem. Phys.

6. H. Utiyama and Y. Tsunashima, Appl. Optics, 9, 1330 (1970); see also H. Utiyama, N. Sugi, M. Kurata, and M. Tamura, Bull. Inst. Chem. Res., Kyoto Univ., 46, 77 (1968).

7. C. Wippler and G. Scheibling, J. chim. Phys., 51, 201 (1954).

8. J. A. Harpst, A. L. Kransa, and B. H. Zimm, Biopolymers, 6, 595 (1968).

9. G. Meyerhoff, U. Moritz, and R. L. Darskus, Polym. Letters, 6, 207 (1968).

10. P. Debye, D. Chu, and H. Kaufmann, J. Polym. Sci., Part A, 1, 2387 (1963).

11. D. McIntyre, J. Mazur, and A. M. Wims, $J$. Chem. Phys., 49, 2887 (1968).

12. J. Mazur, D. McIntyre, and A. M. Wims, $J$. Chem. Phys., 2896 (1968).

13. T. E. Smith and D. K. Carpenter, Macromolecules, 1, 204 (1968).

14. T. Matsumoto, M. S. Thesis, Osaka University, 1970. 